\title{
Amazonie, la forêt qui cache la prairie
}

The Amazon, the forest that hides the pasture

René Poccard, Amaury Bendahan, Soraya Carvalho, Livia Navegantes, Laura Ferreira, Vania Vazfr, Sophie Plassin et Jean-François Tourrand

\section{(2) OpenEdition}

Journals

Édition électronique

URL : https://journals.openedition.org/tc/7453

DOI : $10.4000 /$ tc. 7453

ISBN : 0248-6016

ISSN : 1952-420X

Éditeur

Éditions de l'EHESS

Édition imprimée

Date de publication : 1 mai 2015

Pagination : 146-161

ISBN : 9782713224874

ISSN : 0248-6016

Référence électronique

René Poccard, Amaury Bendahan, Soraya Carvalho, Livia Navegantes, Laura Ferreira, Vania Vazfr,

Sophie Plassin et Jean-François Tourrand, « Amazonie, la forêt qui cache la prairie », Techniques \& Culture [En ligne], 63 | 2015, mis en ligne le 18 décembre 2018, consulté le 29 septembre 2022. URL : http://journals.openedition.org/tc/7453; DOI : https://doi.org/10.4000/tc.7453 


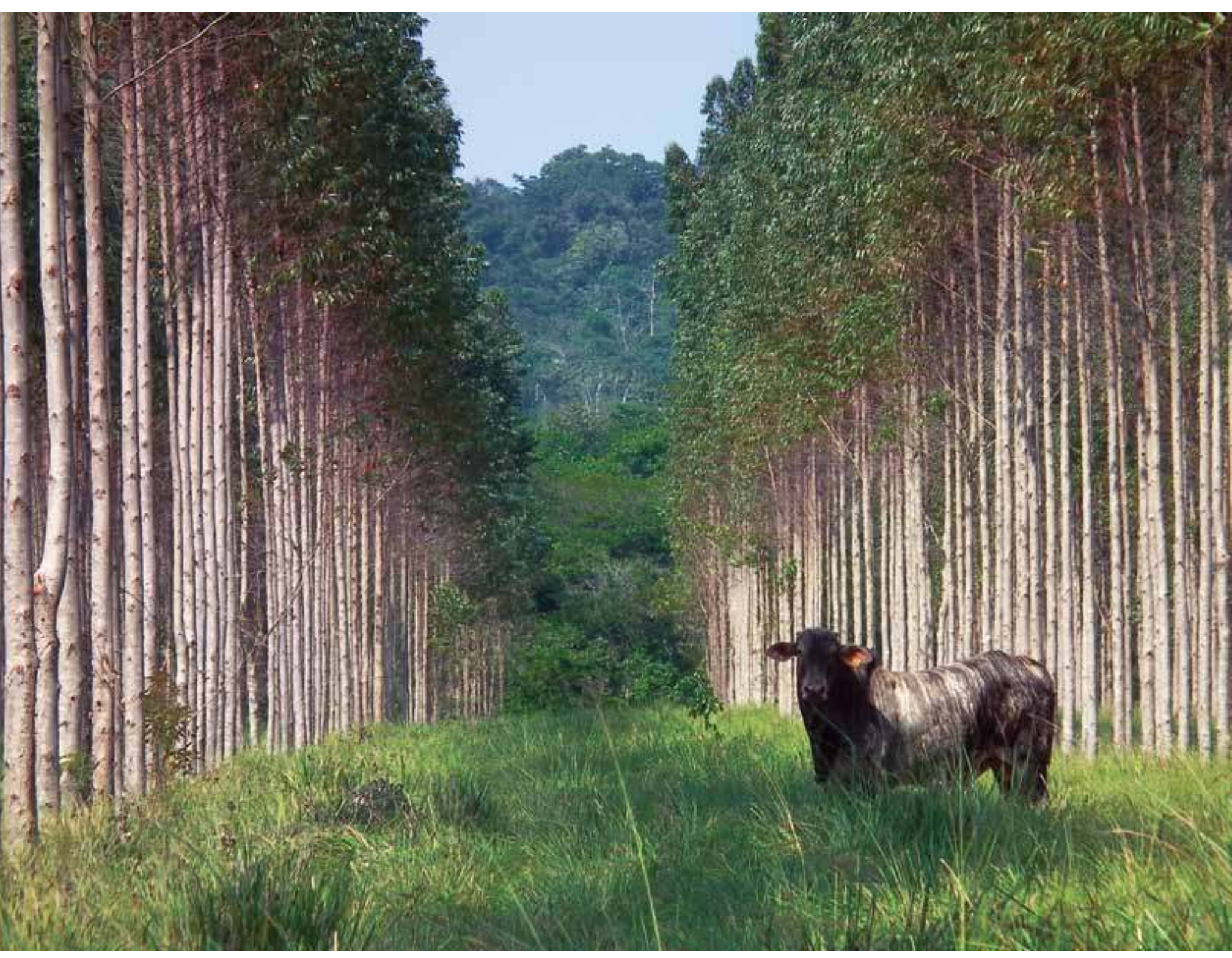

๑ R. René Poccard-Chapuis 


\section{AMAZONIE, LA FORÊT QUI CACHE LA PRAIRIE}

L'Amazonie est plus connue pour sa forêt que pour ses prairies: on l'imagine peuplée d'Indiens plutôt que de vachers. Pourtant, élevage bovin et pâturages sont au cœur de son histoire récente, des bouleversements qui la traversent depuis 50 ans, et dont la déforestation est la partie la plus visible (Sayago \& al., 2004). Loin des clichés véhiculés par les médias internationaux, cet article pose trois questions pour éclairer cette énigme des pâturages amazoniens: dans quelle origine culturelle s'enracine cette dynamique d'expansion des pâturages au détriment du massif forestier? Quels mécanismes agronomiques spécifiques - et quels risques pour les éleveurs - conditionnent cette production fourragère équatoriale? Comment ces prairies peuvent-elles devenir des atouts pour les territoires amazoniens et leurs habitants face aux changements globaux?

\section{Les troupeaux bovins, fer de lance de la colonisation Brésilienne}

\section{L'élevage et la frontière}

Coloniser de nouveaux espaces en y installant des troupeaux bovins est une stratégie pluriséculaire d'expansion du territoire brésilien, depuis l'arrivée des Portugais, face aux occupants indigènes (Deffontaines, 1957). Lactuelle dynamique amazonienne n'est, de

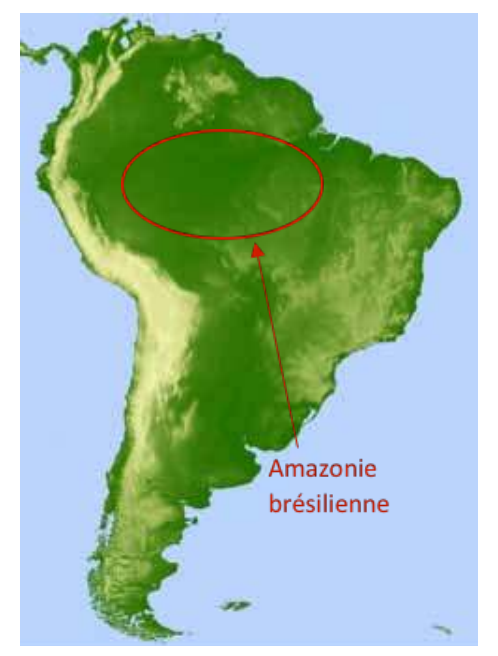


ce point de vue, que la continuité locale d'un processus national que les sertanejos ont initiée dès le $\mathrm{xvI}^{\mathrm{e}}$ siècle dans le Nordeste pour subvenir aux besoins des plantations littorales. Puis ce furent les gauchos dans les pampas du Sud, avant les bandeirantes et autres coroneis dans les savanes du Brésil central. Politiques publiques aidant, chaque cycle de développement du pays provoque ainsi l'occupation de nouveaux espaces pastoraux, dans un mouvement permanent d'avancée de la fronteira, autour de populations spécifiques d'éleveurs pionniers (Théry \& Mello, 2004).

Il existe deux types de frontières au Brésil. L'une, classique et administrative, est fixée par négociations internationales ou affrontements militaires. Mais le territoire qu'elle démarque n'est que partiellement occupé par la société moderne brésilienne, le reste étant aux mains des sociétés indigènes peu ou mal «intégrées». À l'interface se trouve cette "fronteira », territoire marginal et mouvant de colonisation par l'élevage, composante essentielle d'un pays en permanente construction (Monbeig, 1952). Outre des ressources naturelles et agricoles, le Brésil contemporain y a notamment puisé son esprit de conquête, une partie de la structure logistique du territoire, et une culture de l'élevage qui marque fortement l'identité de chaque région, y compris celles aujourd'hui très industrialisées. La déforestation peut se concevoir comme un produit culturel du Brésil contemporain.

Poussée par les troupeaux, la fronteira était historiquement guidée par l'accès aux ressources fourragères et les pistes de bétail (Deffontaines, 1971). Dans un pays très peu accidenté, les éleveurs, ces conquérants civils de nouvelles terres agricoles, ont ainsi progressé au gré des cours d'eau mais surtout des formations herbacées qu'ils rencontraient: steppes, prairies ou savanes, suivant les latitudes (Vaz, 2013). La forêt était alors un obstacle.

\section{Objectif Amazonie}

Au milieu du xx siècle, l'expansion territoriale a trouvé un nouveau souffle, sous l'impulsion de politiques publiques promues par Getulio Vargas et ses successeurs, adeptes du capitalisme d'État dont ils firent du Brésil un champion. Le contrôle du territoire jusqu'à sa frontière politique, par son occupation effective et la valorisation de ses ressources, est devenu une priorité nationale. Ainsi fut organisée la « Marche vers l'Ouest » pour occuper les immenses savanes en direction du Paraguay. Cette migration organisée a achevé la conquête des Cerrados, y compris vers le Nord, aux lisières du massif amazonien.

C'est alors qu'à la fin des années cinquante, Juscelino Kubitschek puis les militaires au pouvoir dans la nouvelle capitale Brasilia, orchestrèrent la conquête de l'Amazonie. Ils assumèrent pleinement la stratégie historique, formulée dans un slogan fameux: « conquérir l'Amazonie par la patte du bouf».

\section{La fièvre de la déforestation}

Une fois encore, la clé de la frontière est entre les mains des éleveurs, en quête de bons pâturages. Le verrou des espaces forestiers a sauté quand ont été mises sur le marché des graines de graminées fourragères pérennes, à semer sur une terre fertilisée par le brûlis. Changement fondamental dans l'histoire pastorale brésilienne, les éleveurs ont pu produire la ressource alimentaire des troupeaux, et ne plus se limiter à une cueillette sur parcours naturels. D'obstacle, la forêt est devenue une ressource, une garantie de fertilité: il suffisait de la brûler pour y cultiver un pâturage, et faire paître les bovins. 
Dès lors les portes de l'Amazonie sont enfoncées par les éleveurs, dépassant les visions politiques de Brasilia. La pluviométrie élevée et constante, les sols apparemment fertiles, l'immensité surtout, promettent une production fourragère, voire agricole, faramineuse. En outre on y découvre des ressources minérales immenses, laissant présager l'éclosion d'un nouveau cycle local de développement. La société des éleveurs -

conquérants, enthousiasmée comme jamais, se tourne massivement vers le Nord, avant même la percée de la route Belém - Brasilia voulue par Kubitschek, en 1960. Celle-ci donnera au mouvement migratoire une ampleur unique dans l'histoire du pays.

C'est le début d'une marée verte, celle des pâturages, qui submerge l'enfer vert, celui de la forêt fermée et hostile aux yeux des colons (Veiga \&t al., 2004). Initialement, les prairies sont installées à la périphérie méridionale du massif amazonien, où d'immenses fermes engraissent les veaux nés dans les zones de savanes: la qualité nutritionnelle des pâturages cultivés et sempervirents, est largement supérieure à celle des parcours naturels, pauvres et secs plus de 6 mois par an. Labondance de cette offre fourragère sans précédent, combinée au coût dérisoire de la terre et de la main-d'œuvre, assure des coûts de production inférieurs aux autres bassins d'élevage nationaux (Margulis, 2003). Bovins et fromages sont commercialisés par camion vers les grands marchés nationaux, en particulier ceux du Nordeste, où la production amazonienne supplante définitivement celle des savanes.

Rapidement des fronts pionniers s'enfoncent vers le cœur du massif sur plusieurs centaines de kilomètres, que ce soit de façon spontanée sous la houlette des réseaux d'éleveurs comme à Sao Félix do Xingu, ou de façon planifiée par le gouvernement fédéral comme sur la Transamazonienne.

Attirés par l'aubaine d'une production bovine abondante et bon marché, les industries de la viande et du lait investissent en Amazonie pour s'y tailler des bassins d'approvisionnement. Des filières complètes, articulées autour d'industries modernes, s'installent sur ces fronts pionniers, qu'elles structurent et dynamisent pour étendre encore les pâturages, produire à moindres coûts des bovins et du lait. Les arbres disparaissent des paysages, au profit des herbacées, formant des prairies continues sur des centaines de kilomètres. Le pâturage est un rouleau compresseur qui désintègre la forêt. Rien ne semble pouvoir le stopper, tant sont solides les chaînes d'intérêts entre acteurs locaux (Mertens \& al., 2002). Tous trouvent leur compte dans cette mer de Brachiaria brizantha, qui à la fin des années quatre-vingt-dix couvre 70 millions d'hectares, la plus grande monoculture au monde, où paissent alors 50 millions de bovins.

\section{Le feu : première étape de l'installation des pionniers}

IL permet « d'ouvrir » la future exploitation agricole, c'est-à-dire de faire reculer la forêt pour dégager des surfaces arables. Celles-ci sont, dans le cas de petites fermes familiales, d'abord cultivées en riz, manioc ou haricots autoconsommés. Ensuite un pâturage est semé, comme ici au premier plan, pour une série de fonctions propres aux fronts pionniers, territoires précaires et isolés par excellence. Dans les grandes exploitations, cette étape agricole n'a pas lieu, le pâturage est semé directement après le brûlis, sur un sol encore fertile, avec donc de meilleurs résultats agronomiques. Le feu est ensuite utilisé régulièrement pour lutter contre les recrus forestiers. La culture agricole des pionniers est ainsi nettement polarisée par le brûlis sous toutes ses formes. Aujourd'hui avec l'assèchement local du climat, résultant des déforestations massives, le feu s'étend par accident et ravage en saison sèche des propriétés entières, voire de grandes portions de territoire. Mais pour s'affranchir du feu, c'est toute la culture agricole, avec les calendriers, outils, savoirs associés, qui doit évoluer. 


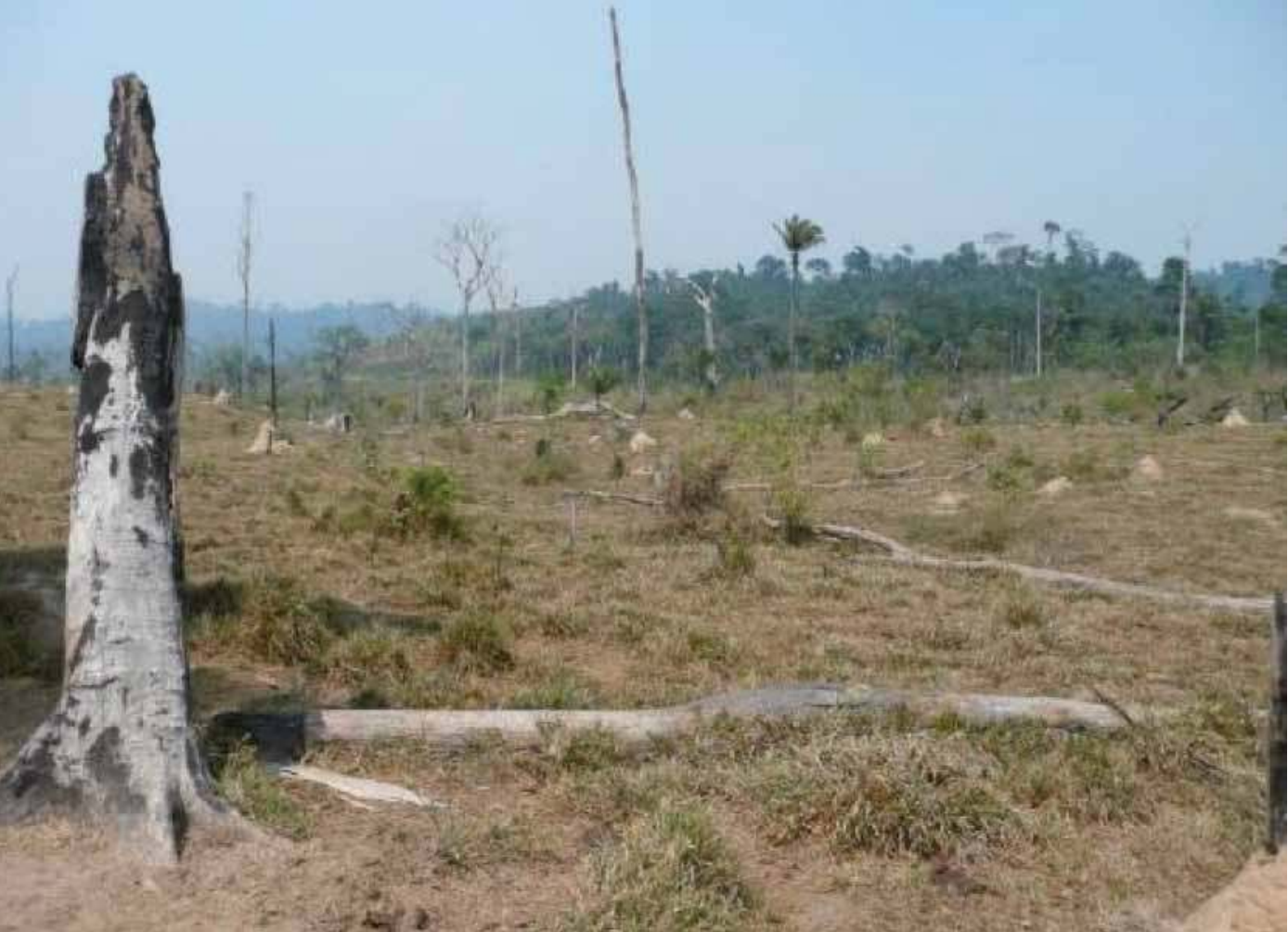

(๑) R. René Poccard-Chapuis
Pendant les premières années après le brûlis initial, le sol est jonché de troncs et souches, qui rendent difficile toute activité agricole en dehors de l'élevage. Le coût du dessouchage est un des principaux freins à la mécanisation des systèmes de production, qui permettrait pourtant de réduire la pression sur la forêt en viabilisant des activités moins consommatrices d'espaces. L'objectif du déforesteur étant avant tout foncier, on voit ici au premier plan que la couverture fourragère est mal formée,

dans ce cas la perte de nutriments du sol perdure: c'est le processus de dégradation des pâturages, qui concerne environ 50 millions d'hectares en Amazonie.
Les bovins élevés en Amazonie sont des zébus, ici de la race Nelore, importée d'Inde dès le XIXe siècle. Trois vachers peuvent s'occuper sur toute l'année d'environ 1000 têtes de bovins: ces races à viande demandent très peu d'interventions humaines, tout comme la graminée Brachiaria brizantha. Entre les étapes de naissage, d'élevage et d'engraissement, les troupeaux de vaches, veaux, broutards circulent d'une ferme à l'autre, parfois sur des centaines de kilomètres, comme ici sur la route de São Félix do Xingú. 
Les années 2000 ont marqué un tournant historique, celui de la « déforestation zéro », l'arrêt probablement définitif de la fronteira au Brésil. En effet, pour mieux entrer dans la nouvelle ère qui s'ouvre, de protection de l'environnement et « d'économie verte », le pays de Lula se pose en champion grâce à deux symboles forts: l'énergie propre (biocarburants tirés de la canne à sucre et du palmier à huile) et la conservation de la forêt amazonienne. Entre 2005 et 2012, Brasilia impose à l'Amazonie un arsenal de politiques publiques rigoureuses et parvient enfin à bloquer la marée verte (Maia \& al., 2011).

L'impact local est énorme: la frontière est bloquée, certes, mais aucun modèle alternatif de développement ne le remplace. Les pâturages ne se conçoivent plus, ni ne se gèrent, comme une arme colonisatrice. Les éleveurs et leurs réseaux doivent inventer une nouvelle trajectoire de valorisation de ces prairies qui couvrent une surface équivalente à celle de la France et de la Belgique réunies (Vaz \& al., 2012).

Ce défi est agronomique, mais aussi territorial et identitaire.

\section{Agronomie des pâturages amazoniens: entre dégradation et intensification écologique}

Même si l'exubérance de la végétation laisse croire le contraire, les sols d'Amazonie sont souvent très peu fertiles, pauvres en matière organique. Sous l'Équateur, la productivité végétale s'appuie sur un équilibre spécifique du système sol-plante, garantissant la circulation rapide des nutriments, depuis les feuilles jusqu'aux racines et inversement. Construire et préserver cet équilibre est un défi agronomique que l'Amazonie impose à ses occupants.

La structure de la forêt amazonienne montre la voie. Elle développe sur quarante mètres de hauteur une épaisse couverture végétale, qui protège le sol de l'agressivité des rayons solaires et des eaux de pluies. En haut, la canopée capte la lumière solaire et les eaux pluviales pour une activité métabolique maximum; en bas un fin humus couvre le sol où court un dense réseau de racines pompant les nutriments issus de la décomposition permanente des feuilles et autres débris végétaux. Entre les deux étages, la matière organique circule sans pertes. Rien ne se perd, rien ne se crée, tout se transforme... très vite, avec efficience.

Qu'en est-il du pâturage? Certes brûler la forêt - 400 tonnes de matière sèche par hectare - est une opération désastreuse dans ce cycle de la matière organique (Blanfort \& al., 2011). La combustion projette ces matériaux dans l'atmosphère sous forme de gaz, équivalant en Amazonie brésilienne à $18 \%$ des émissions de $\mathrm{CO}^{2}$ du pays entier. Mais après cette coupe à blanc, un nouveau circuit fermé de nutriments peut s'installer, ou au contraire l'appauvrissement organique se poursuivre: cela dépend des pratiques de gestion que l'éleveur instaure, et donc de son projet d'élevage et des objectifs qu'il se donne. Quatre pionniers d'Amazonie Orientale nous aident à mieux comprendre ces liens entre circulation des nutriments et culture d'élevage.

Miguel est un éleveur mais surtout un vendeur de terre. Pour lui le pâturage sert avant tout à imprimer dans le paysage les limites spatiales de sa propriété, et à valoriser son capital foncier: un hectare de pâturages se vend entre 5 et 10 fois plus cher qu'un hectare 
années cinquante. Son aventurier de père l'ayant envoyé étudier tandis que lui « affrontait la forêt », il a connu la ville mais ne s'est pas plu à São Paulo. Il utilise maintenant ses connaissances, pour vivre avec sa famille d'un élevage dont il cherche à maximiser les bénéfices sur le temps long. Travailler la fertilité naturelle du sol est un exercice fondamental à ses yeux, d'autant qu'il n'a pas les moyens d'utiliser des engrais chimiques. Perfectionniste, il ne gère pas ses pâturages, il les couve. Un vrai spectacle. Dans ses prairies, Cassio est parvenu à condenser sur 50 centimètres de hauteur les mécanismes de recyclage de la matière organique que la forêt développe sur 40 mètres de hauteur. Sa canopée, c'est le tapis des feuilles sommitales de chaque touffe herbacée, bien serrées, convertissant parfaitement la fantastique énergie solaire de l'Équateur en production de biomasse fourragère. Ce tapis joue aussi le rôle de parapluie pour freiner l'impact et le ruissellement des pluies. En dessous, un épais tapis de feuilles mortes, non pâturées, se décompose et alimente une couche d'humus fraîche et humide, où micro et macrofaune fonctionnent à plein pour aérer le sol, transformer végétaux et minéraux en nutriments, permettre aux eaux pluviales de s'infiltrer sans éroder. L'équilibre du système est d'autant plus robuste qu'il est régulé par une pression de pâturage mesurée et adaptée à chaque parc. Peu de ligneux parviennent à concurrencer les Brachiaria dans de si bonnes conditions. L'entretien est ponctuel, par sarclage, et d'ailleurs Cassio épargne souvent quelques arbustes, qui en grandissant offrent de l'ombre aux bovins et équilibrent leur diète alimentaire. Cassio vit bien de son élevage, d'autant que la génétique de ses bovins, des zébus Nelore sélectionnés, est très performante et convertit au mieux la formidable ressource fourragère qu'il entretient. Son seul vrai problème est le feu accidentel, qui parcourt régulièrement les savanes voisines, et pourrait ruiner cet équilibre agronomique. L'assèchement local du climat, provoqué par les déforestations régionales massives, le désole et il regarde avec envie vers l'Ouest, où la forêt est plus massive et les pluies constantes, près de l'Iriri... là-bas se trouvent Miguel et ses fils, qui auront de l'or entre les mains, si seulement ils apprenaient à gérer leurs pâturages!

Manoel est plutôt un cultivateur pour qui l'élevage est avant tout une épargne. Son pâturage est un compte en banque où il place ses économies sur pied. Celles-ci grandissent au rythme des mises bas, Manoel vendant le moins de bêtes possible. Comme Miguel, comme ses voisins, il a tout quitté pour chercher en Amazonie une redistribution des cartes, loin des sécheresses et famines qui ravagent son Ceará natal. Échappé de la misère, son projet est avant tout de nourrir sa famille, d'élever ses enfants dans la dignité. C'est pourquoi il a adhéré au programme de réforme agraire, et s'est vu attribuer en 198025 hectares de forêt dans la région de Marabá, dans une commune nouvelle au nom évocateur: Eldorado do Carajás. Habitué à la culture sur brûlis dans des steppes buissonnantes semi-arides, cette grande forêt est pour lui une magnifique promesse de champs fertiles: elle produit beaucoup de cendres, sur des sols profonds et sans cailloux. Son riz, son manioc, ses haricots et son maïs y poussent très bien; tous les deux ans il cultive un nouvel hectare pris sur la forêt, et transforme son ancien champ en pâturage en y semant Brachiaria brizantha. À force d'économie, de travaux temporaires dans les grandes fermes voisines où il nettoie les pâturages, il est parvenu à acheter quelques vaches, et est fier de voir son troupeau grandir. Outre le prestige social lié à l'élevage, ce capital sur pied le protège désormais des coups durs, lui permettra d'assurer l'installation de ses enfants à la ville pour suivre le collège et le lycée. Mais Manoel connaît mal l'agronomie de ses pâturages, pas plus que l'équilibre précaire entre sols et plantes qui prévaut en Amazonie. Sa culture technique est celle du feu, et non de la matière organique. Il ne 
perçoit pas que son troupeau grandit plus vite que sa ressource fourragère. En arrivant au bout de sa forêt, ne pouvant plus étendre ses prairies, la charge animale devenant excessive, Manoel a provoqué un surpâturage chronique. Les bovins broutent à l'excès les graminées, râpent la prairie mettant à nu un sol qui s'érode, donnant aux graines d'adventices l'accès à la lumière jusqu'ici monopolisé par les graminées. Celles-ci germent et prennent rapidement le dessus sur des Brachiaria affaiblies, dans des sols appauvris: l'offre fourragère est anéantie en deux ans, une brousse remplace la prairie, c'est la crise du pâturage. Comme ses compagnons d'Eldorado, Miguel doit vendre massivement ses bovins amaigris au moment où les prix sont défavorables. Il perd beaucoup. Mais contrairement à quelques-uns de ses voisins, il garde courage et ne vend pas sa terre. Quel autre point de chute trouverait-il dans cette région pionnière? La ville autant que la fronteira l'inquiètent. Peu ont cette sagesse, et les favelas se multiplient dans tous les pôles urbains d'Amazonie. Désormais orphelin de ses forêts - combustibles, Manoel cherche pour sa terre un nouveau système de production qui puisse durer. Arboriculture? Fruiticulture? Sylvopastoralisme? Un peu des trois? Cette crise lui a ouvert les yeux, il se pose de nouvelles questions, mais ce ne sera pas facile de récupérer la productivité de ses sols. Il incarne l'enjeu social et technique de sa région.

Bernardo, éleveur à Paragominas est un entrepreneur qui a de la chance. Il a investi au bon endroit, au bon moment, et a su travailler sa terre. Son père et lui cultivaient du café au nord de Rio, dans une petite exploitation. Il n'a pas voulu partir vers l'Amazonie à l'époque, au contraire de ses cousins. Mais lorsqu'il hérite en 2002, il vend tout et se mue en investisseur éclairé: bien informé par sa famille, il choisit une région post-pionnière, Paragominas, où les services et institutions sont déjà relativement développés, où les filières d'intrants sont présentes grâce aux cultivateurs de soja qui s'installent aussi. Il choisit 400 hectares vallonnés, impropres à l'agriculture mécanisée donc moins chers, mais proches d'une route goudronnée donc parfaite pour un élevage laitier. Et il investit massivement dans la réforme de ses pâturages: chaulage, apports de phosphates, implantation d'une graminée nouvelle beaucoup plus productive que Brachiaria brizantha, le Mombaça. Cette graminée (Panicum maximum) est cependant beaucoup plus difficile à conduire, elle ne tolère pas les erreurs de gestion ou de charge, et demande une attention fine de l'éleveur. Bernardo approche les agronomes et zootechniciens de l'Embrapa, l'institut brésilien de recherche agronomique. Avec eux il apprend les pratiques de pâturage intensif tournant, qui permet de tripler la charge animale grâce à une alternance rapide entre-temps de pâture et temps de repos, dans des parcelles de petite taille. Grâce à sa génétique bovine adaptée à l'Équateur, des zébus Gir croisés avec des taurines hollandaises (le Girolanda), Bernardo produit 8000 litres de lait par hectare et par an sans autres compléments alimentaires que du sel minéral. Ses pâturages sont en équilibre, ses graminées très vigoureuses combattent seules les adventices: son coût de production est très bas. Il est devenu un notable et un «modèle » pour les éleveurs de la région, car il a su valoriser les qualités de son environnement agro-écologique et social, tout en évitant ses pièges et fragilités, mettant cet ensemble au service d'une intensification raisonnée et efficace. Tout cela sans savoir lire ni écrire. Son nouvel objectif est de devenir un pionnier de la connaissance, explorer d'autres façons de faire pour mieux exploiter les qualités spécifiques des agroécosystèmes amazoniens. L'idée est d'augmenter encore la production, ou de réduire ses coûts, sans artificialiser son système ni le rendre dépendant d'intrants extérieurs. Il pense notamment à l'introduction de plantes légumineuses, arborées, arbustives et herbacées, qui toutes peuvent améliorer la diète et le confort des bovins, la digestibilité 
et la résistance des graminées aux sécheresses estivales de plus en plus récurrentes, fertiliser le sol, tout cela grâce à l'azote contenu dans l'atmosphère. C'est un projet d'intensification écologique, un futur possible pour l'Amazonie.

Mais Bernardo est une exception, et pour lancer un tel mouvement d'intensification à large échelle c'est tout le territoire qui doit s'y mettre. Or les territoires aussi ont des trajectoires mouvementées, en Amazonie.

\section{Territoires en herbe. Trajectoires des fronts pionniers amazoniens, perspectives pour un élevage bovin durable}

La trajectoire classique de construction de territoires au Brésil, après l'ère indigène, débute donc ici comme ailleurs sous la patte du bœuf: couverts de pâturages nos fronts amazoniens ne sont que des territoires en herbes.

L'Amazonie vient de stopper sa phase de défriche, où le principal outil agricole était le feu: elle émerge tout juste d'un néolithique agraire (Poccard-Chapuis \&t al., 2011). Il lui reste à inventer une nouvelle agriculture équatoriale, qui soit à la fois intensive car il ne fait pas de doutes que ses millions de nouveaux hectares agricoles seront fortement sollicités pour nourrir le monde de demain; mais qui soit aussi efficiente dans l'usage des ressources naturelles, abondantes mais fragiles sous l'Équateur, et devenues rares dans le monde. De fait, l'environnement amazonien est un patrimoine - une préoccupation- pour toute l'humanité. C'est ce qui donne tant de puissance internationale à la politique amazonienne conservationniste lancée par Lula.

\section{Construire sur des cicatrices}

Face à cette ambition de durabilité, l'Amazonie part de loin. Nos quatre pionniers en ont expliqué la dimension agronomique, mais ils auraient aussi beaucoup à dire sur la dimension humaine, sociale. La ruée vers la terre est d'abord une lutte pour la terre, une histoire que les acteurs ont souvent écrite avec du sang (Schmink \& Wood, 1991). Miguel pourrait nous parler des affrontements armés où il coordonnait des petits « posseiros » à la recherche de terres libres, contre les grands propriétaires et leurs pistoleiros aux intérêts contraires. Dans sa région, le travail esclave était une réalité, les massacres d'ouvriers agricoles aussi, une balle revient moins chère qu'un salaire à verser. Manoel a connu beaucoup d'assassinats, voire de massacres comme à Eldorado en 1996. Ils visent aussi les représentants syndicaux ou religieux qui défendent les plus humbles, face aux
Dans ce pâturage de la dernière variété de Panicum maximum, cultivar Mombaça, la digestibilité et la vigueur des touffes impressionnent les éleveurs. Par contre, pour gérer correctement la pression de prélèvement par les bovins, ou pression de pâturage, il est nécessaire d'observer finement et régulièrement le comportement et l'état de la plante, et des bovins. La règle apparemment simple de faire rentrer puis retirer les bovins d'un parc au bon moment, est en réalité complexe car multicritère, devant être optimisée à l'échelle de toute la ferme et non d'un seul parc, en fonction des événements climatiques, commerciaux, sanitaires, agronomiques et zootechniques. On comprend que la formation des éleveurs est une étape importante de l'intensification et de la préservation des forêts en Amazonie. l'élevage de précision trouvera dans la région un bon potentiel d'applications. $\grave{A}$ l'arrière-plan, la forêt ne recule plus, elle marque désormais le paysage. 


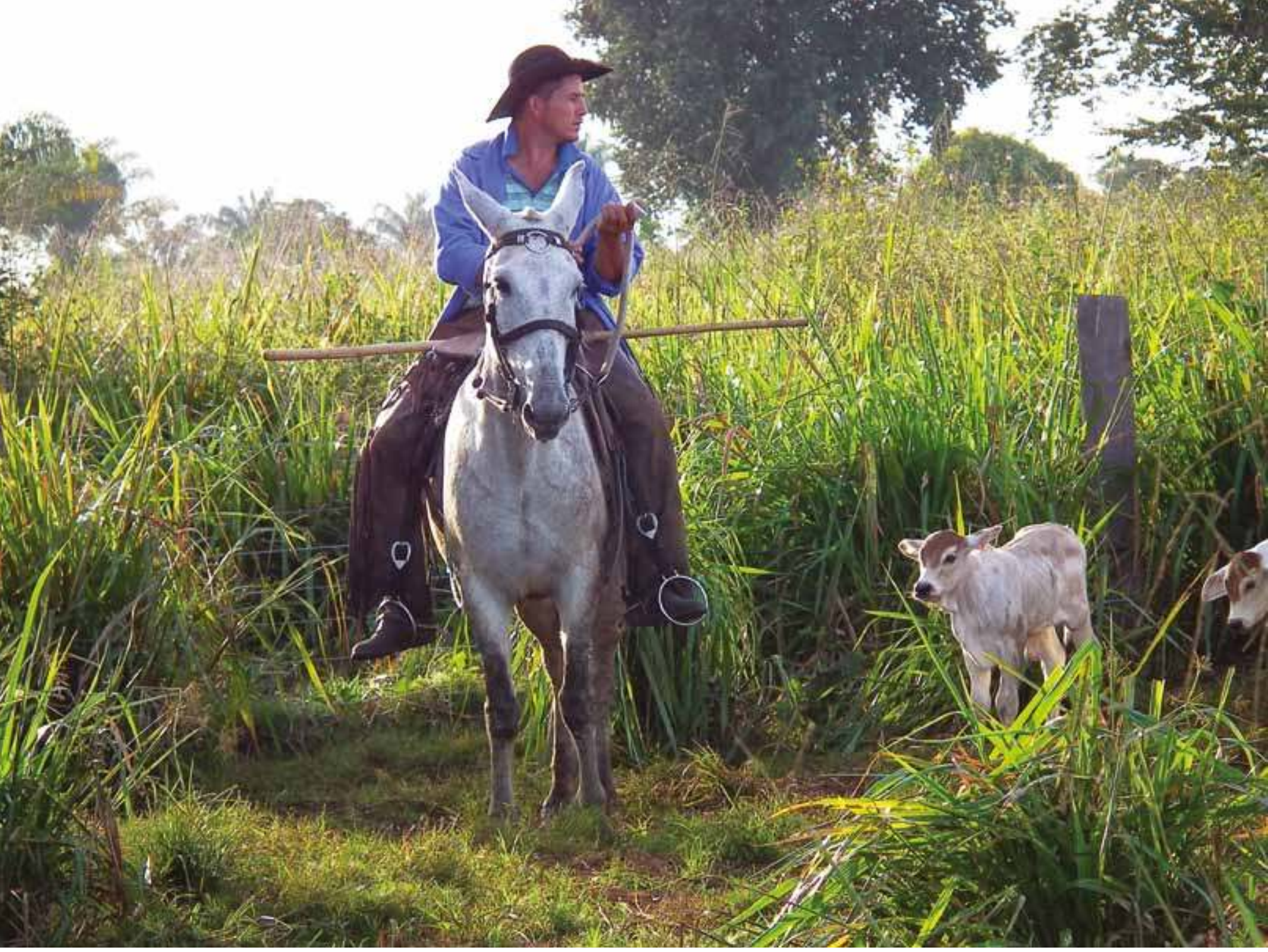

@ R. René Poccard-Chapuis

Le cheval, ou ici la mule, est un outil de travail essentiel au vacher, pour se déplacer et contrôler son troupeau. II s'agit toutefois de métiers peu attrayants pour les jeunes, alors que pour adopter des techniques plus efficientes, les élevages doivent intensifier le recours à une main-d'œuvre efficace et formée aux techniques agronomiques, zootechniques, écologiques. Les motos et surtout les quadricycles se développent d'ailleurs rapidement dans les fermes qui en ont les moyens.

À l'arrière-plan, la hauteur d'herbe témoigne de l'impressionnante production fourragère des prairies amazoniennes, quand elles sont bien gérées et couvrent le sol.

ambitions effrénées que la fronteira fait naître mais qu'elle ne maitrise pas. Cassio, lui, expliquera que la violence est contagieuse et qu'il est difficile de distinguer les bons et les méchants, citant des grands propriétaires envahis, chassés ou tués eux aussi. L'Amazonie est alors un immense Monopoly rural, où l'unique règle du jeu est le rapport de force. Violence et corruption en découlent. L'État, si actif pour lancer la colonisation, a très vite perdu le contrôle de la partie (Tourrand \& al., 2007).

Mais ce feu social tend à disparaître, comme son équivalent agronomique, avec l'arrêt de la frontière. Ainsi Bernardo vit en paix, parce qu'il est arrivé récemment, et parce qu'il a choisi un territoire plus mûr, où s'inventent déjà les bases d'un développement post-pionnier. Sa ville Paragominas était pourtant surnommée Parago-balle il y a 10 ans! C'est sur des cicatrices sociales encore vives que se construisent les nouvelles coordinations territoriales. 


\section{Nouvelles organisations sociales, entre territoires et multinationales}

La gestion minière des ressources naturelles dans des systèmes d'élevage extensifs constituait une solution pour les pionniers, car la structure du territoire de fronteira ne permettait pas autre chose (voir encadré). De fait, ce ne sont pas les solutions techniques qui manquent. Certaines sont très simples: mieux gérer la hauteur d'herbe et les temps de repos, par exemple. D’autres plus radicales, variétés de graminées, génétique bovine, gestion intensive des pâturages, utilisation de paillis, légumineuses, intégration agriculture-élevage, sylvopastoralisme... Il n'existe pas de limites purement techniques pour produire de façon durable de la viande et du lait sous l'Équateur.

Mais comment ces alternatives peuvent-elles sortir des tiroirs de la recherche, ou des parcelles d'innovateurs exceptionnels? Leur mise en œuvre dans les fermes amazoniennes demande des coordinations sociales qui dépassent le seul horizon des éleveurs, et dont la description pourrait donner lieu à un livre plutôt qu'un paragraphe. L'implication de l'État est l'élément le plus visible, tant ont brillé par leur absence ses trois pouvoirs, législatif, exécutif et judiciaire. Un code forestier a bien été voté en 2012, mais il reste peu précis, guère opérationnel sur le terrain pour orienter clairement les décisions des éleveurs. Décentraliser vers les communes ce travail de précision, ces responsabilités environnementales et foncières semble la seule voie possible, face à l'immensité et la diversité de la région. Les décideurs locaux peuvent en outre traduire à leur échelle une législation nationale rendue trop complexe par les tractations politiques entre groupes parlementaires opposés, entre lobbys écologistes et filières agro-exportatrices. Mais cela suppose l'émergence et la consolidation d'institutions locales cohérentes entre elles, bien formées, loin d'une primitive - pour ne pas dire sauvage - ruée vers la terre. Outre la politique, intensifier une ferme nécessite le concours de banques, qui doivent développer des lignes de crédits adaptées, mettre au point un système d'assurances, lequel ne peut fonctionner sans l'octroi de titres de propriété foncière aux pionniers, sans une assistance technique efficace, elle-même formée et dotée de ressources et équipements adéquats pour épauler des éleveurs enclavés au bout de plusieurs heures de pistes chaotiques. Les syndicats et actions collectives sont aussi incontournables, tant pour faciliter l'accès à des services, intrants ou marchés, que pour négocier avec leurs vis-à-vis dans ce qui constituera un projet territorial de développement. Tout cela doit être planifié et consigné dans des zonages, des plans communaux d'aménagement et d'occupation des sols. L'intensification de l'élevage sur les fronts pionniers a besoin d'acteurs et de projets territoriaux, mais tout reste à faire.

Pourtant, l'enjeu des incitations, encadrements et apprentissages dans les territoires, c'est la durabilité sociale. L'urgence est de permettre aux populations d'éleveurs de se maintenir dans le territoire, se développer par l'intensification et la diversification de l'usage des sols, plutôt que de vendre la terre et tenter une nouvelle chance, dans les villes cette fois. Peu d'éleveurs amazoniens pourraient parvenir seuls à s'adapter aux nouvelles contraintes, notamment environnementales. À tant vouloir protéger la forêt, on rend vulnérable les pionniers.

Comme souvent au Brésil, les territoires dépourvus de projets cohérents sont exposés aux seules règles du marché, dont la puissance est amplifiée en Amazonie par l'attractivité croissante de ces vieux pâturages (Pacheco \& Poccard-Chapuis, 2012). L'Amazonie attire les fonds de pensions et autres spéculateurs de la production agricole, aimantés par les potentiels agronomique, énergétique et foncier des zones déjà déforestées, l'ensemble 
étant valorisé par les ports, chemins de fer et canaux que Brasilia construit pour intégrer ce nouvel Eldorado dans la mondialisation des échanges. Une banque de São Paulo possède déjà plus d'un million d'hectares, répartis en une myriade de fermes acquises entre la Terre du Milieu, Redençao et le fleuve Araguaia. Pour ces apôtres globaux de la rente financière à deux chiffres, un bon territoire amazonien est un territoire que l'on pourra, au gré des cotations en bourses, couvrir d'eucalyptus, de soja ou de palmier à huile... Leur vision du défi agronomique se limite à l'artificialisation chimique de l'équilibre sol-plante: engrais, désherbants et pesticides. Le territoire est vu comme un outil de production, que l'on spécialise, dont on se sépare dès qu'on trouve mieux, dès que la ressource s'épuise ou que le marché évolue. Les Pampas argentine et uruguayenne, les savanes brésiliennes du Goiás, montrent les limites sociales et environnementales de ce modèle économique en cluster.

\section{$\&$}

Pour la plupart, les territoires pionniers amazoniens se sont construits autour des filières bovines, autour de cet élevage extensif à la fois salvateur pour des pionniers isolés de toute autre forme de développement économique et social, mais aussi destructeur des ressources naturelles emblématiques et prometteuses pour les générations à venir. L'élevage en Amazonie s'est construit en opposition avec la forêt, les pâturages ne pouvant s'implanter que sur ses cendres, leur productivité dépendant d'une bonne maîtrise des ligneux envahissants.

Aujourd'hui cette déforestation disparait, promouvant de nouvelles synergies entre arbres et pâturages (photo 9). Conduits en association à l'échelle des parcelles, des paysages, des territoires, ils contribuent à reconstituer et maintenir des ressources devenues rares, comme l'eau et la matière organique dans les sols, en plus de rendre des services écosystémiques comme le stockage de carbone ${ }^{2}$, ou encore améliorer le confort animal et la productivité zootechnique.

Les 50 ans d'avancée de l'élevage sur la forêt ont placé entre les mains des populations amazoniennes une surface agricole utile de plus de 500000 kilomètres carrés. Grâce aux formidables capacités des agro-écosystèmes équatoriaux, elles peuvent, si elles parviennent à conduire les bons projets territoriaux, développer un élevage et une agriculture écologiquement intensifs, où la ressource naturelle est mieux exploitée et entretenue, à l'exemple de l'eau et la matière organique des sols. L'enjeu reste donc de savoir comment cette recherche d'écoefficiences peut être assimilée par les acteurs locaux, pour devenir un objectif de développement territorial dans les bassins d'élevage. Chercheurs, éleveurs, décideurs, devront trouver les réponses ensemble. C'est ce que la Patagonie n'a pas su faire, cent ans en arrière, comme l'explique F. Coronato dans ce numéro.

En d'autres termes, on demande aux éleveurs amazoniens et aux acteurs de leurs territoires, de passer du néolithique à l'agriculture du xxi siècle en quelques décennies. Cela tombe bien, une caractéristique des fronts pionniers est le dynamisme et la capacité d'adaptation de ses occupants. Les pâturages, les arbres et les éleveurs amazoniens sont en tout cas bien au cœur du chantier de la durabilité dans ce nouvel ordre agricole et écologique mondial. 
Ce travail a été conduit dans le cadre du projet de recherches MOUVE financé par l'Agence nationale de la recherche (projet ANR-2010-STRA-005-01).

Auteurs: René Poccard (CIRAD), Amaury Bendahan (Empresa Brasileira de Pesquisa Agropecuária), Soraya Carvalho (Université Fédérale du Parà), Livia Navegantes (UFPa - Université Fédérale du Pará), Marcelo Thalês (MPEG), Laura Ferreira (UFPa - Université Fédérale du Pará), Vania Vaz (Université de Brasilia), Sophie Plassin (CIRAD), Jean-François Tourrand (CIRAD).

\section{NOTES}

Photo d'ouverture: Loin de ses fonctions initiales orientées vers le foncier, le pâturage vise aujourd'hui la performance non seulement agronomique, mais aussi écologique. Il s'agit d'optimiser l'usage des ressources naturelles, de s'organiser dans l'espace pour aménagement des secteurs de retour à une forêt naturelle, et d'autres à des systèmes sylvopastoraux. Mais partout l'arbre reprend une nouvelle place dans les fermes.

1. Les terres appartiennent en réalité à l'État. Ce sont des forêts publiques, mais exposées à une appropriation privée par le mouvement de colonisation. C'est l'objet de la « lutte pour la terre » décrite notamment par les sociologues Octavo Ianni (1978), puis Marian Schmink et Charles Wood (1992).
2. Blanfort \& al. montrent que les vieux pâturages amazoniens, de plus de 20 ans, peuvent stocker plus de carbone dans le sol que la forêt tropicale, grâce aux populations de micro-organismes dans les horizons pédologiques profonds.

\section{POUR CITER CET ARTICLE}

René Poccard, Amaury Bendahan, Soraya Carvalho \& al. 2015 Amazonie, la forêt qui cache la prairie. In Dupré, L., Lasseur, J. \& R. Poccard-Chapuis Pâturages Techniques E Culture 63: 146-161.

\section{RÉFÉRENCES}

Blanfort, V., Doreau, M., Huguenin, J., \& al., 2011 Impacts et services environnementaux de l'élevage en régions chaudes, Productions animales, 24 (1): 89-112.

Deffontaines, P., 1957 L'Introduction du bétail en Amérique Latine. Les cahiers d'Outre-Mer, 10 : 5-22, Bordeaux 1957.

Deffontaines, P., 1971 Le Rôle de l'élevage dans la régionalisation de l'espace au Brésil. In La régionalisation de l'espace au Brésil, Anais do congresso de 20 - 22 novembre 1968. Paris: Éditions du CNRS; 46-56.

Ianni, O., 1978. A luta pela terra. Petropolis: Vozes.

Maia, H., Hragrave, J., Gomez, J., \& al. 2011 Avaliação do plano de ação para prevenção e controle do desmatamento na Amazônia legal. Documento do Instituto de Pesquisa Econômica Aplicada. IPEA: Rio de Janeiro.

Margulis, S., 2003 Causas do desmatamento na Amazônia brasileira. Banco Mundial: Brasilia.

Mertens, B., Poccard Chapuis, R., Piketty, M.G. \& al. 2002 Crossing spatial analyses and livestock economics to understand deforestation processes in the Brazilian Amazon: the case of Sao Félix do Xingu in South Para. Agricultural Economics 27 (3) : 269-294.

Monbeig, P, 1952 Pionniers et planteurs de São Paulo. Paris : Armand Colin. 
Pacheco, P. \& Poccard-Chapuis, R., 2012 The complex evolution of cattle ranching development amid market integration and policy shifts in the Brazilian Amazon. Annals of the Association of American Geographers, 102 (6) : 1366-1390.

Poccard-Chapuis, R., Bonaudo, T., Lossouarn, J., 2011 Élevage, filières et territoires en régions chaudes, Productions animales 24 (1): 129-144

Sayago, D., Tourrand, J.-F., Bursztyn, M., 2004 Amazônia: Cenas e cenários. Brasilia: Universidade de Brasilia. Seminário sobre Monitoramento Estratégico das Transformações Ambientais (META). 1,2002-06-03/2002-06-14, Brasília.

Schmink, M., Wood, C., 1992 Contested frontiers in Amazônia. New York: Columbia University Press.

Théry, H., Mello, N. A., 2004 Atlas du Brésil. Atlas do Brasil: Disparidades e dinâmicas do território, EDUSP.

Tourrand, J.-F., Valarié, P., Dias de Oliveira, J.-R., 2007 Monopoly Amazonien, Cahiers Agricultures 16 (5): 423-427.

Vaz, V., Abreu de Carvalho, S., Barbosa, T., \& al. 2012 A pecuaria na agenda ambiental da amazônia brasileira: Percepções e representações dos atores locais. In Organizado por Jalcione Almeida, Cleyton Gerhardt, Sônia Barbosa Magalhães (eds). Contextos rurais e agnda ambiental no Brasil: Práticas, políticas, conflitos, interpretações. Dossiê 3. Belem: Rede de Estudos Rurais, 65-90.

Vaz, V., 2013 A formação dos latifúndios no sul do Estado do Pará: Terra, Pecuária e desflorestamento. Brasilia: Université de Brasília (Thèse de doctorat au Centre de Développement Durable).

Veiga, J.-B., Tourrand, J.-F., Piketty, M.G. \& al. 2004 Expansão e trajetória da pecuária no Pará. Brasilia: Universidade de Brasilia.

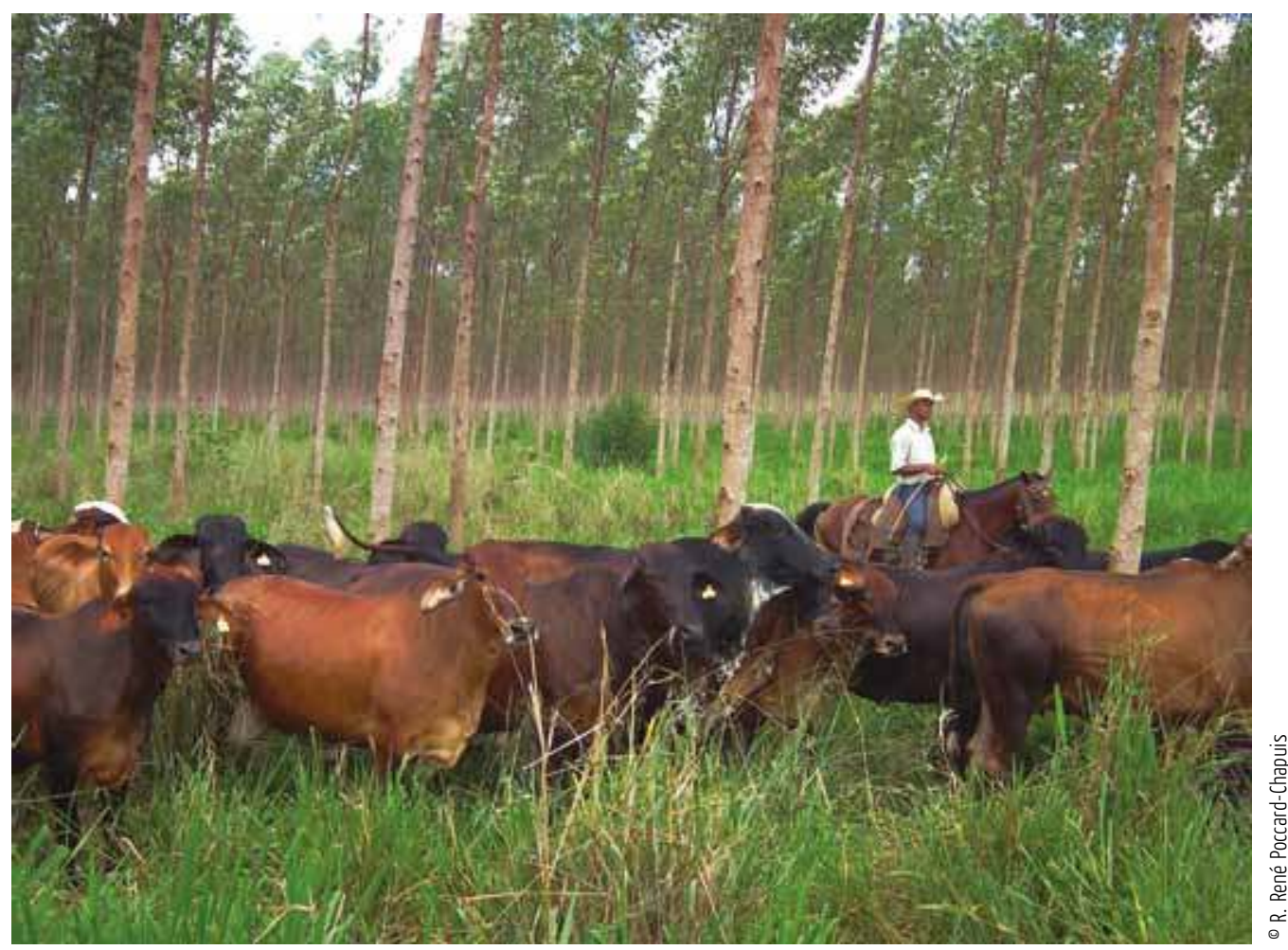

Prise en saison sèche, cette photo illustre les bénéfices multiples des pratiques visant à préserver l'eau dans les sols. Le bon enracinement de la graminée, semée avec un engrais, combinée à l'ombre produite par les eucalyptus, largement espacés dans la prairie, permettent de maintenir une biomasse verte toute l'année. Grâce à cela, les troupeaux maintiennent des indices de performances zootechniques constants dans l'année, évitant le traditionnel infléchissement en saison sèche. Les risques d'incendie sont limités, les troncs servent de poteaux pour des barrières sur mesure, et surtout le confort thermique permet d'utiliser des croisements avec des races européennes, plus productives pour le lait (ici des vaches croisées zébu Gir / taurin Holstein) et la viande. Loin d'être une contrainte, la meilleure gestion des ressources naturelles, ici hydriques, est au contraire une source de meilleures performances: c'est le principe de l'écoefficience. 


\section{RÉSUMÉ}

Amazonie, la forêt qui cache la prairie. L'Amazonie brésilienne est plus connue pour sa forêt que pour ses prairies. Celles-ci poursuivent pourtant, depuis cinq décennies de colonisation par la patte du bœuf, une expansion sans précédents dans le monde de pâturages cultivés, au détriment de la couverture forestière. Le long de fronts pionniers de déforestation, les éleveurs avancent et occupent les terres jusqu'alors publiques ou indigènes, amorçant l'émergence de nouveaux territoires en herbes.

Les auteurs éclairent cette Amazonie des pâturages, méconnue, sous trois angles complémentaires. Ils expliquent en premier l'origine culturelle de l'expansion actuelle des pâturages, en la rattachant à la notion de frontière agricole, propre au Brésil depuis sa découverte par les navigateurs portugais. Le deuxième aspect est relatif aux mécanismes agronomiques qui conditionnent cette production fourragère équatoriale, la rendant à la fois attractive, risquée, changeante. Enfin, le lien aux territoires est explicité, montrant comment autour des pâturages naissant se construisent des territoires en herbes, qui pourront après cette phase initiale de conquête foncière se diversifier et consolider leurs institutions.

Une dynamique nouvelle se fait jour actuellement, le pâturage dégradé devenant une réserve de terres arables pour alimenter le monde dans les prochaines décennies, et enrichir les investisseurs qui suivent les pionniers. C'est l'entrée de la chimie dans les pâturages et l'artificialisation des agroécosystèmes.

Ainsi l'article s'attache à lire les liens entre évolutions des pâturages et des sociétés, ce point de vue permettant de conclure sur les conditions sociales d'émergence de systèmes d'intensification écologique.

\section{ABSTRACT}

The Amazon, the forest that hides the pasture. The Brazilian Amazon Region is best known for his forest for its pasture. Yet their expansion is permanent, after five decades of a cattle ranching colonization process, something unique in the rangeland world. Along deforestation frontier, farmers are going on, they occupy public or indigenous land, beginning the emergence of new region.

The authors explain the Amazonian pasture dynamic, misunderstood, using three complementary point of view. They explain first the cultural origin of the current expansion of pastures, linking with the concept of agricultural frontier, strong in Brazil since its discovery by Portuguese navigators. The second aspect relates to the agronomical mechanisms that determine such equatorial forage production, making it both attractive, risky and changing. Finally, they specify the synergies with regional development, showing how new territories are been built by the grass, forage, cattle and farmers, and how this initial phase of land conquest is a base for diversify economics and strengthen the local institutions.

À new dynamic is emerging currently. The degraded pasture becomes a reserve of arable land to feed the world during next decades, and generate incomes for investors who follow the pioneers. This mean the entrance of chemical incomes in pasture and agroecosystems. Thus, the article attempts to read the links between pastures expansion and society development; this point of view allows a conclusion about social conditions for occurrence of ecological intensification systems.

\section{MOTS CLÉS}

Amazonie, éleveurs, fronts pionniers, pâturages, déforestation, intensification écologique

\section{KEYWORDS}

The Amazon, cattle farmer, pioneer front, pasturelands, deforestation, ecological intensification 